\title{
A numerical scheme for non-local aggregation with non-linear diffusion and approximations of social potential
}
F. Georgiou ${ }^{1}$

$$
\begin{aligned}
& \text { N. Thamwattana }{ }^{2} \quad \text { B. P. Lamichhane } \\
& \text { J. E. F. Green }
\end{aligned}
$$

(Received 11 December 2020; revised 19 February 2022)

\begin{abstract}
Aggregations abound in nature, from cell formations to locust swarms. One method of modelling these aggregations is the non-local aggregation equation with the addition of degenerate diffusion. In this article we develop a finite volume based numerical scheme for this style of equation and perform an error, computation time, and convergence analysis. In addition we investigate two methods for approximating the non-local component.
\end{abstract}

DoI:10.21914/anziamj.v62.16056, (C) Austral. Mathematical Soc. 2022. Published 2022-03-07, as part of the Proceedings of the 19th Biennial Computational Techniques and Applications Conference. ISSN 1445-8810. (Print two pages per sheet of paper.) Copies of this article must not be made otherwise available on the internet; instead link directly to the DOI for this article. 


\section{Contents}

1 Introduction

C243

2 Numerical scheme

C245

3 Results

C248

4 Conclusion

C252

\section{Introduction}

Aggregations abound in nature, from cell formations [9] to locust swarms [1]. While there are a variety of techniques in modelling these phenomena, one popular technique is the non-local aggregation equation. First proposed by Mogilner and Edelstein-Keshet [12], it is a conservative mass system of the form

$$
\frac{\partial \rho(x, t)}{\partial t}+\nabla \cdot([-\nabla Q(x) \star \rho(x, t)] \rho(x, t))=0,
$$

where $\mathrm{Q}(\boldsymbol{x})$ is a scalar function of $\boldsymbol{x} \in \mathbb{R}^{\mathfrak{n}}$ and called the social interaction potential, $\rho(x, t)$ is the density of species in question, and $\star$ is the convolution operator in space. For this model the existence and stability of swarms has been proven [12], and both travelling wave solutions [12] and analytic expressions for the steady states [1] have been found. However, in this form the maximum density of steady state solutions grow unbounded with the addition of mass. It was thus further extended to include non-linear local repulsion in the form

$$
\frac{\partial \rho}{\partial t}+\nabla \cdot(\rho[-\nabla(Q \star \rho)-\gamma \rho \nabla \rho])=0,
$$

which leads to compact and bounded solutions [13]. Finally, linear diffusion is added either for modelling or simulation purposes to give

$$
\frac{\partial \rho}{\partial t}+\nabla \cdot(\rho[-\nabla(\mathrm{Q} \star \rho)-\gamma \rho \nabla \rho])-\mathrm{D} \Delta \rho=0 .
$$


It is this style of equation that we study in one dimension. While initially used for single populations, the model has been further adapted to consider a variety of interacting species and populations $[9,14,6]$.

The difficulty in simulating (1) arises from the non-local interaction term. While it is possible to directly calculate this term [7], it is more efficient to use Fourier transforms [2,3]. The key to using Fourier transforms is the convolution theorem [5], which states that under suitable conditions the Fourier transform of a convolution of two functions is equal to the point-wise product of their individual Fourier transforms, that is,

$$
\mathscr{F}\{\mathbf{f} \star \mathbf{g}\}=\mathscr{F}\{\mathbf{f}\} \cdot \mathscr{F}\{\mathbf{g}\},
$$

where $\mathscr{F}$ represents the Fourier transform and $f$ and $g$ are two integrable functions on $\mathbb{R}^{n}$ (we also denote the inverse Fourier transform as $\mathscr{F}^{-1}$ ). We also use another property of convolutions given by

$$
\nabla(\mathrm{f} \star \mathrm{g})=(\nabla \mathrm{f}) \star \mathrm{g}=\mathrm{f} \star(\nabla \mathrm{g}) .
$$

As $\mathrm{Q}$ does not depend on time, (2) and (3) allow us to calculate $\mathscr{F}\{\nabla \mathrm{Q}\}$ once at the beginning of the simulation and then be used throughout.

In this article we use and compare two methods to find $\mathscr{F}\{\nabla \mathrm{Q}\}$. The first is performing a discrete time Fourier transform (DTFT) as done by Topaz et. al. [14], and the second is to define $\nabla Q$ on our domain $x=[0, L]$ in such a way that we can approximate the DTFT using a discrete Fourier transform (DFT) [8]. We begin with a common social potential known as the Laplace potential,

$$
\mathrm{Q}(\mathrm{x})=\mathrm{e}^{\frac{-|x|}{r}},
$$

whose derivative has the DTFT [14]

$$
\operatorname{DTFT}\left\{\frac{\partial}{\partial x} e^{-\frac{|x|}{r}}\right\}=\frac{i \Delta x \sin (\Delta x q)}{r[\cosh (\Delta x / r)-\cos (\Delta x q)]},
$$


Table 1: Definitions of symbols used in numerical scheme at arbitrary cell $i$.

\begin{tabular}{l|l} 
Symbol & Definition \\
\hline$\Delta x$ & spatial size of cells in the $x$ direction \\
$\mathbf{x}$ & vector representing the discretised spatial grid \\
$x_{i}$ & $x$ value of the midpoint of a grid cell \\
$P_{i}$ & approximate function values of $\rho$ \\
$\mathbf{P}$ & vectors representing the discretised function $\rho$ \\
$\mathscr{N}_{i}$ & approximate value of the non-local component of the equation \\
$\mathscr{A}_{i}$ & approximate value of the advective component of the equation \\
$\mathscr{D}_{i}$ & approximate value of the diffusive component of the equation
\end{tabular}

where $\mathbf{q}$ is the frequency domain. The approximation is [8]

$$
\frac{\partial}{\partial x} e^{\frac{-|x|}{r}}= \begin{cases}0 & \text { if } x=0, \\ \frac{1}{r}\left(-e^{-\frac{|x|}{r}}+e^{-\frac{|(L+\Delta x)-x|}{r}}\right) & \text { if } x>0 .\end{cases}
$$

which is then converted to the frequency domain using a DFT. We term (5) the exact DTFT and (6) the approximate.

We begin in Section 2 by introducing a numerical scheme for simulating (1) in one dimension. Then, in Section 3 we look at convergence and computation time for the numerical scheme using both methods of transforming $\nabla \mathrm{Q}$ to the frequency domain and understanding what error is introduced by using the approximate transform (6) versus exact DTFT (5).

\section{Numerical scheme}

We now derive the numerical scheme for (1) in one dimension using a finite volume method (FVM) with a linearised Riemann approximator [10]. For the numerical scheme the terms described in Table 1 are used in relation to an arbitrary cell $i$ with cell boundaries $i \pm \frac{1}{2}$. To begin we approximate the local 
part of the velocity term using a central differencing scheme, giving

$$
-\gamma \rho \frac{\partial \rho}{\partial x} \approx-\gamma \mathrm{P}_{\mathrm{i}} \frac{\mathrm{P}_{\mathrm{i}+1}-\mathrm{P}_{\mathrm{i}-1}}{2 \Delta \mathrm{x}},
$$

at an arbitrary cell $i$. Then, for the non-local component of the velocity term, we use (2) and (3) to turn the convolution component of the advection term into

$$
-\nabla(\mathrm{Q} \star \rho)=\mathscr{F}^{-1}\left\{\mathscr{F}\left\{-\frac{\partial}{\partial x} \mathrm{Q}\right\} \cdot \mathscr{F}\{\rho\}\right\} .
$$

We then approximate the convolution as

$$
-\nabla(\mathrm{Q} \star \rho) \approx \mathscr{N}=\operatorname{real}\left\{\operatorname{ifft}\left\{\mathscr{F}\left\{-\frac{\partial}{\partial x} \mathrm{Q}\right\} \cdot \operatorname{fft}\{\mathbf{P}\}\right\}\right\},
$$

where fft and ifft represent the fast Fourier transform and inverse fast Fourier transform, respectively. We take only the real component of the ifft as any imaginary value will simply be due to error. By combining the local and non-local components and letting

$$
F_{i}=\left(-\gamma P_{i} \frac{P_{i+1}-P_{i-1}}{2 \Delta x}+\mathscr{N}_{i}\right) P_{i}
$$

and using a standard Riemann approximator we estimate the wave-speed at a cell boundary, $i-\frac{1}{2}$, as

$$
S_{i-\frac{1}{2}}= \begin{cases}\frac{F_{i}-F_{i-1}}{P_{i}-P_{i-1}} & P_{i} \neq P_{i-1}, \\ \frac{F_{i}-F_{i-1}}{\Delta x} & P_{i}=P_{i-1},\end{cases}
$$

and the wave size as

$$
W_{i-\frac{1}{2}}=P_{i}-P_{i-1}
$$

We then approximate the advection component using an upwind scheme, giving

$$
\mathscr{A}_{i}=\frac{1}{\Delta x}\left(\max \left\{S_{i-\frac{1}{2}}, 0\right\} W_{i-\frac{1}{2}}+\min \left\{S_{i+\frac{1}{2}}, 0\right\} W_{i+\frac{1}{2}}\right) .
$$


It is worth noting that as we multiply $S_{i-\frac{1}{2}}$ with $W_{i-\frac{1}{2}}$ the denominator of the fraction used to calculate $S_{i-\frac{1}{2}}$ is cancelled out and the only information that is used is the sign of $P_{i}-P_{i-1}$. We therefore simplify (7) and (8) to

$$
\widehat{S}_{i-\frac{1}{2}}=\left(F_{i}-F_{i-1}\right) \operatorname{sign}\left(P_{i}-P_{i-1}\right),
$$

and

$$
\hat{W}_{i-\frac{1}{2}}=\operatorname{sign}\left(P_{i}-P_{i-1}\right)
$$

where

$$
\operatorname{sign}(x)= \begin{cases}-1 & x<0 \\ 0 & x=0 \\ 1 & x>0\end{cases}
$$

giving the upwind scheme for the advection component as

$$
\mathscr{A}_{i}=\frac{1}{\Delta x}\left(\max \left\{\hat{S}_{i-\frac{1}{2}}, 0\right\} \hat{W}_{i-\frac{1}{2}}+\min \left\{\hat{S}_{i+\frac{1}{2}}, 0\right\} \hat{W}_{i+\frac{1}{2}}\right) .
$$

Next, for the diffusion term, we approximate this using FVM as

$$
\mathscr{D}_{\mathrm{i}} \approx \frac{\mathrm{D}}{\Delta \mathrm{x}^{2}}\left(2 \mathrm{P}_{\mathrm{i}}-\mathrm{P}_{\mathrm{i}-1}-\mathrm{P}_{\mathrm{i}+1}\right) .
$$

Combining all the terms we obtain

$$
\mathrm{P}_{i}^{\mathrm{t}+\Delta \mathrm{t}}=\mathrm{P}_{\mathrm{i}}^{\mathrm{t}}-\Delta \mathrm{t}\left(\mathscr{A}_{i}+\mathscr{D}_{\mathrm{i}}\right) .
$$

Finally, for time integration we use the Dormand-Prince method [4]. This is an adaptive Runge-Kutta method where a fourth and a fifth order scheme are calculated simultaneously with the fifth order scheme being used as an error estimate for the fourth order scheme. This error estimate is then used to adapt the time step [11]. This method is used in the MatLaB function ode45. 


\section{Results}

In order to test our numerical scheme of (1) we ran a series of simulations with 64, 128, 256, 512, 1024 and 2048 grid cells. The initial densities are given by $\rho(x, 0)=\max (2+\mu, 0)$ where $\mu$ is some normally distributed noise with mean 0 and standard deviation 1. In addition, one set of initial conditions was created at the lowest resolution and interpolated to higher resolutions to ensure all simulations had the same initial conditions. The spatial domain was $x \in[0,20]$ with periodic boundary conditions (i.e., $\rho(0, t)=\rho(20, t)$ ) and time was run to the pseudo steady state $t=500$.

We used the following social potential for gregarious locust behaviour as it is commonly used in literature $[1,14,6]$,

$$
\mathrm{Q}(\mathrm{x})=\operatorname{R} e^{\frac{-|x|}{r}}-A e^{-|x|} .
$$

We then took both the exact DTFT and approximate transform (given by (5) and (6), respectively). The constants were set as $\mathrm{D}=0.01, \gamma=0.6$, $R=0.25, A=1$ and $r=0.5$. Finally, we let the 2048 grid cells with exact DTFT be our reference solution; a snapshot at times $t=0,0.72,4.80$ and 500 is shown in Figure 1.

The error and computation time is shown in Figure 2, a) and b), respectively. In order to calculate the error, we interpolated our reference solution to each lower resolution (denoted $\mathbf{P}_{\text {ref }}$ ), then for each number of grid cells $(64,128,256,512$ and 1024) and each social potential (approximate and exact DTFT) we calculated our total error as

$$
\epsilon=\left\|\mathbf{P}_{\text {ref }}-\mathbf{P}\right\|,
$$

where $\|\cdot\|$ represents the standard $\mathrm{L}_{2}$ norm. The error is calculated at the pseudo steady state of $t=500$. From this we estimated the average convergence rate as greater than 1.8 for both the exact DTFT and approximate social potentials (1.8829 and 1.9114, respectively), however the approximated social potential has a non-uniform convergence. Computation time was 
Reference simulation (2048 grid points, exact DTFT)
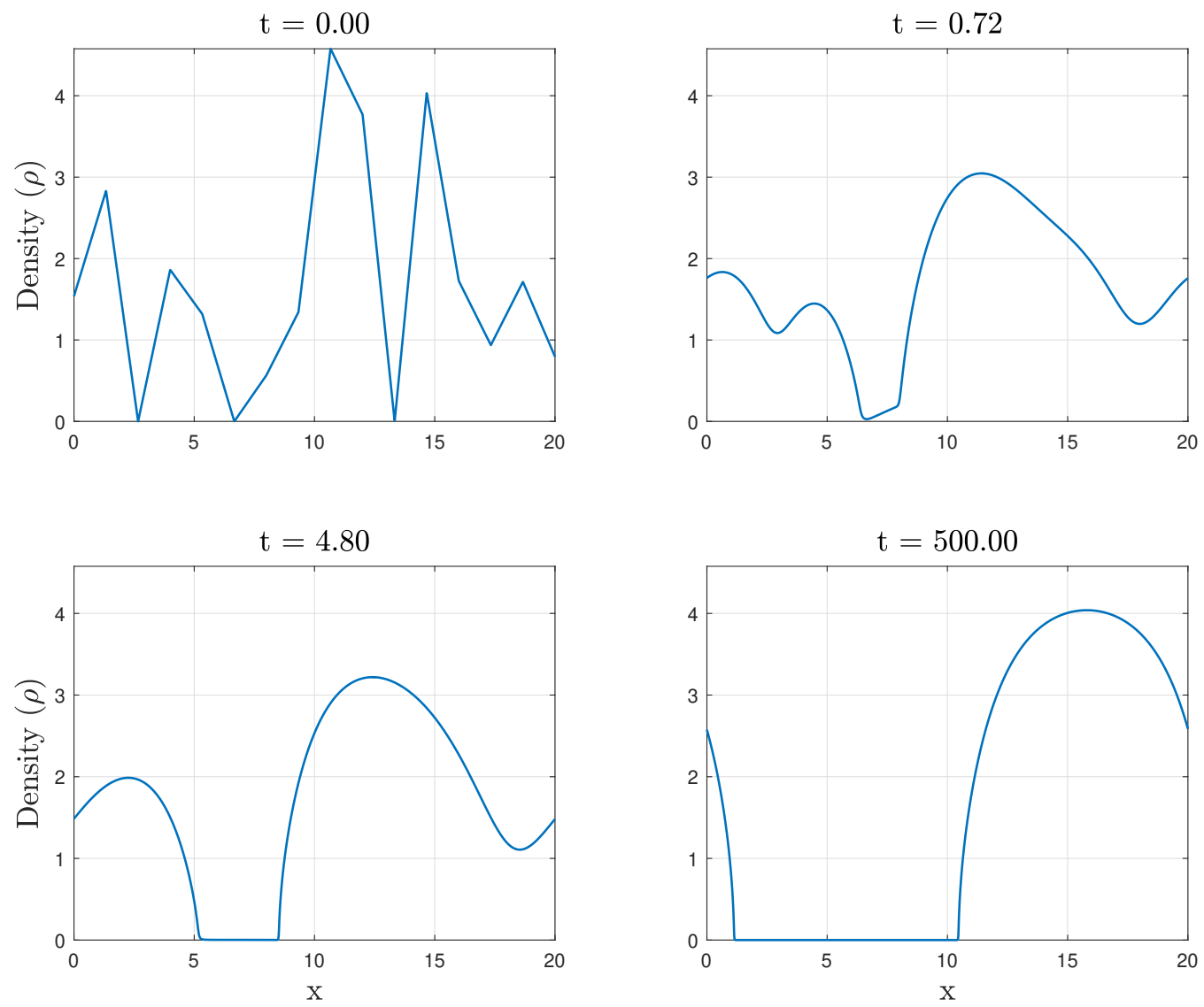

Figure 1: Reference solution of (1) with social potential given by (9) with $\mathrm{D}=0.01, \gamma=0.6, \mathrm{R}=0.25, \mathrm{~A}=1$ and $\mathrm{r}=0.5$ on the periodic domain $x \in[0,20]$. The reference solution uses the exact DTFT given by (5) and 2048 grid cells. 


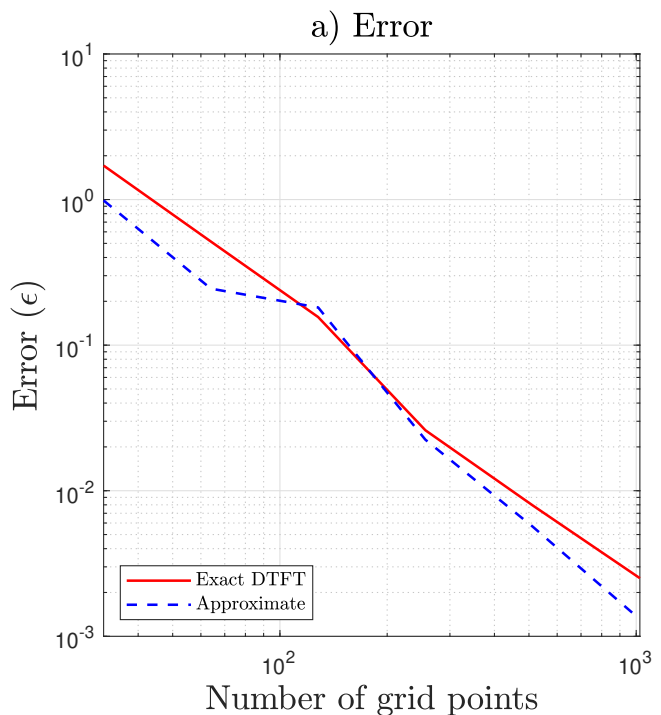

b) Time

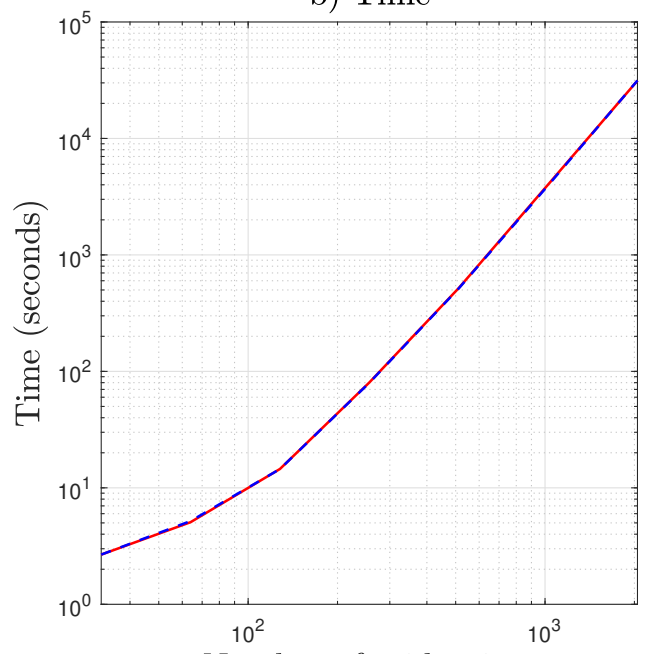

Number of grid points

Figure 2: Numerical Error Estimates $(\epsilon)$ and computation time for $64,128,256,512$ and 1024 grid cells at time $t=500$ for both the exact DTFT and approximated social potentials with $\mathrm{D}=0.01, \gamma=0.6, \mathrm{R}=0.25$, $A=1$ and $r=0.5$.

comparable for both approximated and exact DTFT social potentials. The most startling aspect of this numerical experiment is that for the majority of grid cells the approximation is closer to the reference solution than the exact DTFT.

To test whether the approximation continues to be more accurate than the exact DTFT, we ran simulations with a different set of constants. The constants in (4) were changed to $R=1, A=1$ and $r=0.1$ with everything else set as before. The error and computation time is shown in Figure 3, a) and b), respectively. It is again seen that at certain resolutions the approximation outperforms the exact DTFT. This time the average convergence rate is greater than 1.7 for both the exact DFT and approximate social potentials (1.7512 and 1.8684 , respectively). 
a) Error

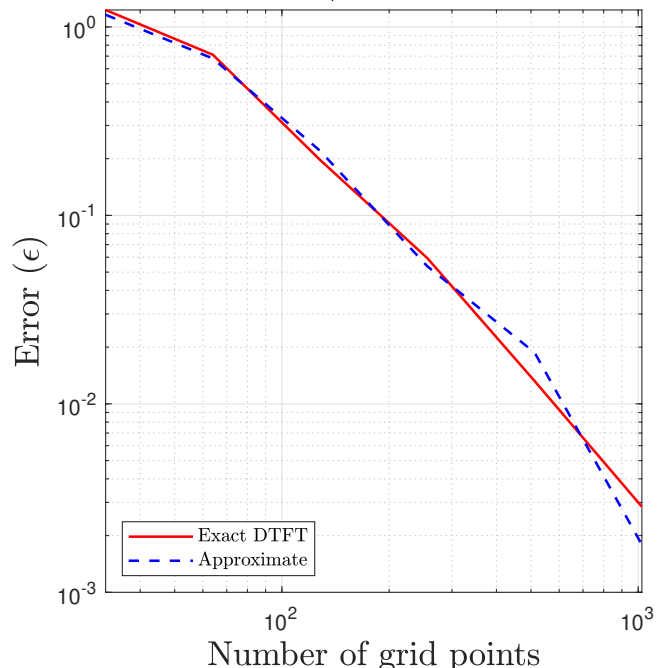

b) Time

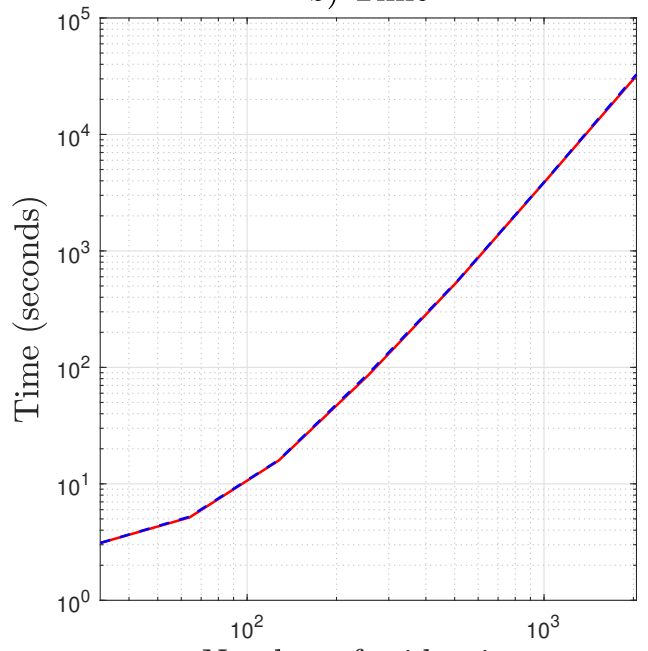

Number of grid points

Figure 3: Numerical Error Estimates $(\epsilon)$ and computation time for $64,128,256,512$ and 1024 grid cells at time $t=500$ for both the exact DTFT and approximated social potentials with $\mathrm{D}=0.01, \gamma=0.6, \mathrm{R}=1$, $A=1$ and $r=0.1$.

In order to better understand this, we turned back to our original values and looked at the relative error between the high resolution (2048 grid cells) exact DTFT of the social potential and the lower resolution exact DTFT and approximate social potentials. We then defined the relative error between social potentials as

$$
\epsilon_{\mathrm{rel}}=\frac{\left\|\mathbf{Q}_{\mathrm{ref}}-\mathbf{Q}_{\mathrm{app}}\right\|}{\left\|\mathbf{Q}_{\mathrm{ref}}-\mathbf{Q}_{\mathrm{ext}}\right\|},
$$

where $\mathbf{Q}_{\text {ref }}$ is the high resolution exact DTFT, $\mathbf{Q}_{\text {app }}$ is the approximation, and $\mathbf{Q}_{\text {ext }}$ is the lower resolution exact DTFT (we also calculate the relative error between simulations in a similar manner). The social potentials, $\mathbf{Q}_{\text {ref }}$ and $\mathbf{Q}_{\mathrm{ext}}$, have been converted back to the spatial domain using a DFT. The results can be seen in Figure 4 (blue line, left axis), with $\epsilon_{\text {rel }}<1$ showing that the estimate is more accurate than the exact DFT for each resolution. While the 


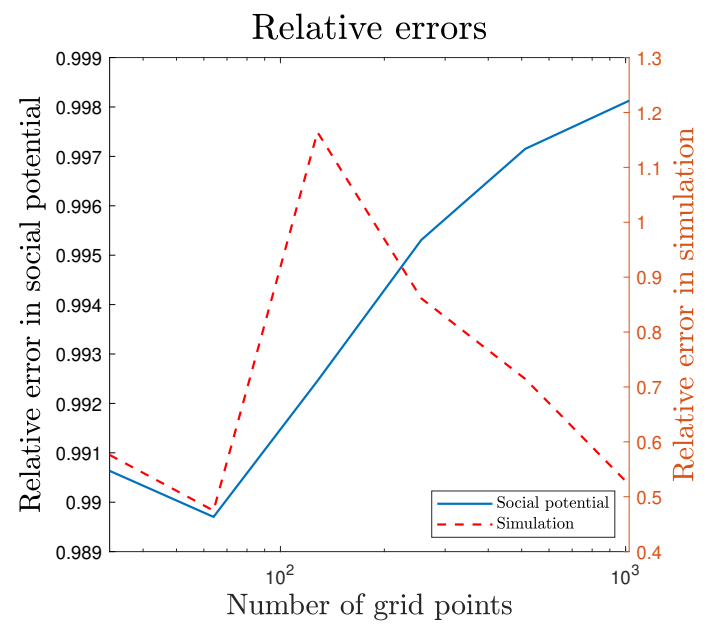

Figure 4: $\epsilon_{\text {rel }}<1$ indicating that the approximation is more accurate than the exact DTFT for each resolution. While the approximation is closer to the reference social potential at each resolution it is not a good predictor of the relative error between simulations (red dashed line, right axis)

approximation is closer to the reference social potential at each resolution it is not a good predictor of the relative error between simulations (red dashed line, right axis).

\section{Conclusion}

In this article we introduced a finite volume based numerical scheme for simulating (1) and tested its accuracy and convergence using a common social potential. In addition, we compared an exact DTFT of the social potential with a method for approximating it and found that under the tested conditions the approximation outperformed the exact DTFT. While more work needs to be done to understand why this is the case, the approximation method appears to work well and could be used for social potentials with difficult to calculate DTFTs. 


\section{References}

[1] A. J. Bernoff and C. M. Topaz. "Nonlocal aggregation models: A primer of swarm equilibria". In: SIAM Rev. 55.4 (2013), pp. 709-747. DOI: $10.1137 / 130925669$ (cit. on pp. C243, C248).

[2] R. Bürger, D. Inzunza, P. Mulet, and L. M. Villada. "Implicit-explicit methods for a class of nonlinear nonlocal gradient flow equations modelling collective behaviour". In: Appl. Numer. Math. 144 (2019), pp. 234-252. DOI: 10.1016/j . apnum.2019.04.018 (cit. on p. C244).

[3] J. A. Carrillo, A. Chertock, and Y. Huang. "A finite-volume method for nonlinear nonlocal equations with a gradient flow structure". In:

Commun. Comput. Phys. 17.1 (2015), pp. 233-258. DOI: 10.4208/cicp.160214.010814a (cit. on p. C244).

[4] J. R. Dormand and P. J. Prince. "A family of embedded Runge-Kutta formulae". In: J. Comput. Appl. Math. 6.1 (1980), pp. 19-26. DOI: 10.1016/0771-050X(80)90013-3 (cit. on p. C247).

[5] J. von zur Gathen and J. Gerhard. Modern computer algebra. 3rd ed. Cambridge University Press, 2013. DOI: 10.1017/CB09781139856065 (cit. on p. C244).

[6] F. Georgiou, J. Buhl, J. E. F. Green, B. Lamichhane, and N. Thamwattana. "Modelling locust foraging: How and why food affects group formation". In: PLOS Comput. Biol. 17.7 (2021), e1008353. DOI: 10.1371/journal.pcbi.1008353 (cit. on pp. C244, C248).

[7] F. Georgiou, B. P. Lamichhane, and N. Thamwattana. "An adaptive numerical scheme for a partial integro-differential equation". In: Proceedings of the 18th Biennial Computational Techniques and Applications Conference, CTAC-2018. Ed. by B. Lamichhane, T. Tran, and J. Bunder. Vol. 60. ANZIAM J. 2019, pp. C187-C200. DOI: 10.21914/anziamj.v60i0.14066 (cit. on p. C244). 
[8] F. Georgiou, N. Thamwattana, and B. P. Lamichhane. "Modelling cell aggregation using a modified swarm model". In: Proceedings of the 23rd International Congress on Modelling and Simulation, MODSIM2019. Vol. 6. 2019, pp. 22-27. DOI: 10.36334/modsim.2019.a1.georgiou (cit. on pp. C244, C245).

[9] J. E. F. Green, S. L. Waters, J. P. Whiteley, L. Edelstein-Keshet, K. M. Shakesheff, and H. M. Byrne. "Non-local models for the formation of hepatocyte-stellate cell aggregates". In: J. Theor. Bio. 267.1 (2010), pp. 106-120. DOI: 10.1016/j.jtbi.2010.08.013 (cit. on pp. C243, C244).

[10] R. J. LeVeque. Finite-volume methods for hyperbolic Pproblems. Cambridge Texts in Applied Mathematics. Cambridge University Press, 2002. DOI: 10.1017/CB09780511791253 (cit. on p. C245).

[11] C. F. Van Loan. Introduction to Scientific Computing: A Matrix Vector Approach Using MATLAB. 1997. URL:

https://www pearson.com/us/higher-education/program/VanLoan-Introduction-to-Scientific-Computing-A-Matrix-VectorApproach-Using-MATLAB-2nd-Edition/PGM215520.html (cit. on p. C247).

[12] A. Mogilner and L. Edelstein-Keshet. "A non-local model for a swarm". In: J. Math. Bio. 38.6 (1999), pp. 534-570. DOI: 10.1007/s002850050158 (cit. on p. C243).

[13] C. M. Topaz, A. L. Bertozzi, and M. A. Lewis. "A nonlocal continuum model for biological aggregation". In: Bull. Math. Biol. 68 (2006), p. 1601. DOI: 10.1007/s11538-006-9088-6 (cit. on p. C243).

[14] C. M. Topaz, M. R. D'Orsogna, L. Edelstein-Keshet, and A. J. Bernoff. "Locust dynamics: Behavioral phase change and swarming". In: PLOS Comput. Bio. 8.8 (2012), e1002642. DOI:

10.1371/journal.pcbi.1002642 (cit. on pp. C244, C248). 


\section{Author addresses}

1. F. Georgiou, School of Mathematical and Physical Sciences, University of Newcastle, Callaghan, NSW 2308, Australia. mailto:fillipe.georgiou@uon.edu.au orcid:0000-0003-4588-5319

2. N. Thamwattana, School of Mathematical and Physical Sciences, University of Newcastle, Callaghan, NSW 2308, Australia. orcid:0000-0001-9885-3287

3. B. P. Lamichhane, School of Mathematical and Physical Sciences, University of Newcastle, Callaghan, NSW 2308, Australia. orcid:0000-0002-9184-8941

4. J. E. F. Green, School of Mathematical Sciences, University of Adelaide, Adelaide, SA, 5005, Australia. orcid:0000-0001-5061-9563

5. J. Buhl, School of Agriculture, Food and Wine, University of Adelaide, Adelaide, SA, 5005, Australia. orcid:0000-0002-7506-6835 